
\title{
25 Research Suare \\ Case Report: Gastric Cancer Combined With Bilateral Ovarian Metastasis In Pregnancy
}

\section{Zhenhua Zhang}

Licang women's Health Department

\section{Gang Lv}

Shandong First Medical University

Mei Liu

Licang women's Health Department

Miaohui Lin

Qingdao Hospital of Traditional Chinese Medicine

Meilin Xie

Qingdao Hospital of Traditional Chinese Medicine

Yinghui Zhao ( $\nabla$ zhaoyinghui1971@outlook.com )

Shandong First Medical University https://orcid.org/0000-0002-1072-2205

Jia Lv

Qingdao Hospital of Traditional Chinese Medicine

\section{Case report}

Keywords: Gastric cancer, Pregnancy, Metastatic ovarian cancer, Ascites

Posted Date: October 18th, 2021

DOI: https://doi.org/10.21203/rs.3.rs-871687/v1

License: (c) (i) This work is licensed under a Creative Commons Attribution 4.0 International License.

Read Full License 


\section{Abstract}

Background: The early manifestations of gastric cancer during pregnancy are non-specific. Ovarian mass and ascites are the main manifestations of late ovarian metastasis.

Case presentation: A case of late gastric cancer with bilateral ovarian metastasis in pregnancy was reported in our study. Cesarean section and full staging of ovarian malignant tumors were performed at $28^{+5}$ weeks of pregnancy, and the prognosis was poor. We performed Cesarean delivery and extracted a vital male newborn of $35 \mathrm{~cm}, 1020 \mathrm{~g}$, Ap score 3 . The newborn is alive and grows normally. Three months later the mother died. The key to improving the prognosis is early diagnosis, and the pre-pregnancy health checkup must be emphasized.

Conclusions: During pregnancy, ultrasound, gastroscopy, MRI and other related examinations should be performed to confirm the diagnosis, and individualized treatment plans should be formulated to maximize the protection of the mother and child's interests.

\section{Background}

In recent years, the incidence of gastric cancer in young people ( $\leq 40$ years of age) has been on the rise [1], more women than men were reported [2], and seriously threaten the health of women of childbearing age. Gastric cancer is usually related to the combined effects of multiple factors such as lifestyle and diet, family susceptibility, and bacterial infections [3]. Ovarian cancer includes primary and metastatic. Metastatic ovarian cancer may originate from any part of the body. However, the metastasis of gastric cancer to the ovary in late pregnancy is relatively rare in clinical practice, and it is often difficult to diagnose early. In this case, a pregnancy woman, with gastric cancer and bilateral ovarian cancer was reported.

\section{Ethics Statement}

This study was approved Qingdao hospital of traditional chinese medicine (Qingdao Haici hospital affiliated to Qingdao University) medical college committee under contract 2021HC01LS020 obstetrics.

\section{Case Presentation}

Young female, 31 years old, 1 pregnancy and 0 birth. She was admitted to the hospital on September 7, 2019 due to abdominal distension and abdominal effusion for half a month. Normal menstruation is regular, the cycle is 28 days. Last menstruation was February 28,2019 . Urine pregnancy test was positive after $30^{+}$days of menopause, morning nausea occurred after 50 days of menopause, and no vomiting occurred since pregnancy. Fetal movement occurred after 4 months. After $6^{+4}$ weeks of menopause, the external hospital ultrasound examination showed early pregnancy, the size of the left ovarian cyst was $4.6 \mathrm{~cm} \times 3.1 \mathrm{~cm}$ and without treatment. Two weeks ago $\left(25^{+2}\right)$, she felt abdominal distension. Color Doppler 
ultrasound found that there was an uneven echo in each of the appendages. There were multiple small cystic echoes, which resembled ovarian echoes. The size of the right side is about $6.6 \mathrm{~cm} \times 3.8 \mathrm{~cm}$, and the left side is about $6.0 \mathrm{~cm} \times 4.4 \mathrm{~cm}$. The left appendix area is cystic, the size is about $4.7 \mathrm{~cm} \times 3.0 \mathrm{~cm}$, and the liquid free dark area is explored in the periphery of liver, spleen, and bilateral iliac fossa. OGTT (Oral glucose tolerance test) is normal, and blood pressure during pregnancy is normal. The liver and kidney function tests were generally normal. Tumor markers CEA $1.24 \mathrm{ng} / \mathrm{ml}$; AFP $356.91 \mathrm{lU} / \mathrm{ml} \uparrow$, CA199 9.24U/ml, CA125 196.8U/ml $\uparrow$. On the day of admission, splenomegaly and large abdominal effusion was found from abdominal ultrasound. A solid medium echo was found in the front of the uterus in the left lower abdomen, with a range of about $8.0 \mathrm{~cm} \times 5.8 \mathrm{~cm} \times 6.4 \mathrm{~cm}$, with clear borders and visible capsules in pelvic ultrasound. The echo is closely related to the anterior peritoneum, with a large amount of fluid in the abdominal cavity, with a maximum depth of about $8.7 \mathrm{~cm}$. Body weight increased by $8 \mathrm{Kg}$ during pregnancy, poor diet, good sleep, and normal stool. She was not exposed to toxic or harmful substances during pregnancy.

Ultrasound examination was performed on September 9, 2019. In the right appendix area, a medium and low echo light cluster was detected, the size was about $11.0 \mathrm{~cm} \times 6.4 \mathrm{~cm}$, the boundary was still clear, and the shape was obviously irregular. CDFI (Color Doppler Flow Imaging) showed rich blood flow signal, RI 0.33. A $7.4 \mathrm{~cm} \times 5.6 \mathrm{~cm}$ heterogeneous mixed acousto-optic cluster was detected in the left appendix. The morphology was regular, the boundary was still clear, multiple cystic echoes were found inside. CDFI showed slightly rich blood flow signal, RI 0.29 . The upper part of the left appendix was closely connected with a single room cystic echo of $5.3 \mathrm{~cm} \times 4.1 \mathrm{~cm}$, with clear capsule and good sound transmission. The deepest pelvic-abdominal effusion is about $12.5 \mathrm{~cm}$. Electronic gastroscopy showed flaky mucosa redness and swelling at the descending junction of the duodenal bulb. The gastric antrum mucosa is rough, red and swollen, with normal peristalsis. The gastric mucosa was rough, and there was an ulcer with a diameter of about $1.0 \mathrm{~cm}$ in the greater curvature of the gastric body (Figure 1). The ulcer had irregular edges, covered with white fur, and the surrounding mucosa was swollen and swelled. Five pieces of tissue were taken for biopsy. The tissue were brittle and hard and easy to bleed, with no abnormalities.

The pathological result of gastroscopy biopsy indicated gastric signet ring cell carcinoma from greater curvature of the stomach was diagnosed. Abdominal plain scan MRI report showed that irregular masses of long T1 and long T2 signal shadows were found in the stomach. DWI presented a slightly higher signal, with still clear edges, and the largest cross-section was about $3.8 \mathrm{~cm} \times 5.1 \mathrm{~cm}$ (Figure 2). The greater omentum was thickened irregularly, presented multiple nodules and strips, with short T1 and long T2 signals, and DWI showed a slightly higher signal (Figure 3). There was a mass of high and low mixed T1 and mixed T2 signal shadows in the bilateral appendage, and DWI showed a high signal. The maximum cross section of the left lesion was about $6.6 \mathrm{~cm} \times 10.0 \mathrm{~cm}$, and the maximum cross section of the right lesion was about $6.4 \mathrm{~cm} \times 7.7 \mathrm{~cm}$, with clear edges. No obvious abnormal swollen lymph nodes were found in the pelvic cavity, and there were no abnormalities. These indicated space-occupying lesions of the gastric body and bilateral ovaries were found, and retroperitoneal lymph nodes and peritoneum might be metastasized. Surgical treatment is not suitable for advanced gastric cancer, and comprehensive tumor 
treatment is recommended. On September 17,2019 , at $28^{+5}$ weeks of pregnancy, cesarean section with transverse incision of lower uterus, Double attachment resection, and intraperitoneal hyperthermic perfusion drainage tube placement were performed. One child was delivered by cesarean section, $35 \mathrm{~cm}$ in length, $1020 \mathrm{~g}$ in weight, and score 3 . Poorly differentiated malignant tumors were infiltrated in both ovarian tissues from postoperative pathology, which suggested the metastatic poorly differentiated adenocarcinoma might originate from the stomach (Figure 4). The pregnancy with gastric malignant tumor and bilateral ovarian malignant tumor, hypoproteinemia and $28^{+5}$ weeks of pregnancy G1P1 were diagnosed after surgery. Targeted chemotherapy, intraperitoneal hyperthermic perfusion chemotherapy, and combined anti-tumor therapy with traditional Chinese medicine were performed after surgery. The patient died more than 3 months after the surgery.

\section{Discussion And Conclusions}

\section{Clinical features}

A series of physiological changes such as loss of appetite, nausea and vomiting during pregnancy are similar to the symptoms of early gastric cancer. Clinicians tend to diagnose that they are normal physiological pregnancy or hyperemesis gravidarum. Even if individual pregnant women have prominent reactions and their symptoms are not relieved for a long time, they are mostly regarded as individuals differences or chronic gastritis, which induce delays in diagnosis. The patient had been waken up in the morning with nausea, no vomiting, and poor appetite for a long time, and had not aroused the doctor's alertness until the appearance of ascites. Compared with the symptoms of the primary tumor, the symptoms of secondary ovarian cancer are often more obvious, such as pelvic mass, abdominal distension, ascites, anemia, weight loss, etc. Clinically, about one-third of ovarian cancer patients are diagnosed with secondary symptoms for the first diagnosis [4]. In order to protect the fetus from being affected, the use of gastrointestinal barium meal, CT and other radiological examinations during pregnancy is limited. Most invasive examinations such as gastroscopy and abdominal puncture are difficult for pregnant women to accept, which often delays the diagnosis. Because the early symptoms of gastric cancer are indistinguishable, and the clinicians are inexperienced in rare cases, the early symptoms are easily overlooked. Once the secondary symptoms appear, the clear diagnosis is mostly late.

\section{Diagnosis}

The types of ovarian tumors are complex and changeable. Therefore, for pregnancy with ovarian tumors, clinicians must consider not only the distinction between benign, malignant and borderline, but also primary and metastatic, which is very difficult. It is clinically differentiated from ovarian physiological cysts, endometriotic cysts, and ovarian epithelial cystadenoma. 
The patient found a unilateral ovarian cyst with a diameter of $<5 \mathrm{~cm}$ since $6^{+4}$ weeks of pregnancy, which was mistaken for a simple ovarian cyst. Ovarian tumors during pregnancy are mostly benign, and about $70 \%$ can resolve spontaneously in the second trimester [5]. The ultrasound examination was not standardized during pregnancy, which delayed the diagnosis and treatment. The detection of the patient found that the ovarian tumor had progressed unilaterally to bilateral, with uneven echoes, and rapid growth in volume in a short time (2 weeks), cystic to solidified, with abundant blood flow signals and low blood flow resistance, and a large amount of ascites, all suggest the possibility of ovarian malignancy [6]. Therefore, ovarian tumors during pregnancy need to be reviewed regularly and closely followed up. The diagnosis should be further confirmed by magnetic resonance imaging (MRI). MRI has been widely used in obstetrics. It has a higher resolution for soft tissue lesions and can more clearly show the relationship between lesions and surrounding organs. It has become another important auxiliary examination method besides ultrasound. It has sensitivity and specificity in the diagnosis of malignant tumors in pregnancy [7].

Gastroscopy biopsy pathological examination is the basis for the diagnosis of this patient, and it is also a key point in the diagnosis of the disease. Although gastroscopy may have adverse effects such as allergic reactions, arrhythmia, local injury and bleeding, premature birth, etc., it is not a contraindication to pregnancy examination. If a pregnant woman has persistent gastrointestinal symptoms for a long time, it cannot be explained by normal pregnancy reactions or malignant lesions indicated by imaging examination, gastroscopy can be performed [8]. Patients should be fully communicated to reduce their concerns and improve compliance. In order to find out the reason, it is necessary to perform invasive cavity puncture. The patient underwent The ultrasound-guided catheter drainage of ascites was performed with strict aseptic operation on the patient. Routine ascites and cytology of ascites sediment are helpful for differential diagnosis.

The detection values of AFP and CA125 in the patient were elevated, but it was little clinical significance because AFP and CA125 also increased during pregnancy. Studies had reported that the changes of CEA, CA199, HE4, and LDH values were not significantly related to pregnancy, and could assist pregnancyrelated ovaries tumor diagnosis[9].

\section{Treatment}

When malignant tumors in pregnancy occur in different periods, the treatment principles are also different. In the first trimester, the mother's health is generally given priority. It is not recommended to continue the pregnancy. It is preferred to actively treat malignant tumors after the termination of the pregnancy. In the second trimester, the severity of the disease and the periods of the gestational week must be considered, then the pregnancy should be continued or terminated. In the third trimester, the gestational time should be appropriately extended to improve the survival probability of the fetus, and it is more common in premature babies [10]. In this case, considering the poor prognosis of the pregnant woman and the patient's expectation for the fetus, the fetal lung maturity was promoted and pregnancy 
was terminated as soon as possible when the survival probability of fetal survival was high. Cesarean section and complete staging of ovarian malignant tumors were performed at $28^{+5}$ weeks of gestation. Follow-up found that the newborn was alive and the growth and development were normal. The treatment of ovarian malignant tumors requires a comprehensive assessment of the tumor (degree of malignancy), pregnant women and their families (the physical condition of the pregnant women, the expectation of the pregnant women and their family members for the fetus), the fetus (gestational age, survival ability), the hospital's critically ill newborns and critically ill pregnant women's ability and other factors $[11,12]$.

\section{Prognosis}

Stage IV gastric cancer in pregnancy with bilateral ovarian metastasis, T3N1M1, has a very poor prognosis, and the patient's survival time is less than 4 months. Changes in hormones and immunosuppressive status during pregnancy promote tumor occurrence and development. The tissue type is highly malignant, which easily spreads to distant lymph nodes and involves vascular [13].

Treatments of radiotherapy and chemotherapy during pregnancy is limited. The patient did not undergo a pre-pregnancy health checkup, which led to the deterioration of the gastric cancer appeared before pregnancy. The key to improving the prognosis of patients is early detection, early diagnosis and early treatment, which is precisely the difficulty faced by clinicians.

\section{Conclusion}

Nowadays, many women of childbearing age are delaying childbirth, and after the implementation of the two-child policy, there has been a significant increase in women (age $\geq 35$ ) who have reborn children, as well as the younger age of onset of tumors, which brings new great challenges to clinicians. Women of childbearing age should strengthen their personal awareness of health during pregnancy, and clinicians should also pay special attention to pre-pregnancy health checkups.

\section{List Of Abbreviations}

OGTT Oral glucose tolerance test

CDFI Color Doppler Flow Imaging

MRI magnetic resonance imaging

\section{Declarations}

\section{Ethics approval and consent to participate}

This study was approved by the Institutional Human Care Committee of Qingdao Hospital of Traditional Chinese Medicine. This report was approved by patient and family members. 


\section{Consent for publication}

The manuscript is approved by patient. The manuscript is approved by all authors for publication.

\section{Availability of data and materials}

No applicable

\section{Competing interests}

The authors declare that they have no competing interests.

\section{Funding}

This work was supported by the National Foundation of Natural Science of China (Grant No. 81602455), the Science Foundation of Shandong Province (Grant No. ZR2013HM033).

\section{Authors' contributions}

$\mathrm{JL}$ and $\mathrm{YZ}$ conceived and designed the study, and critically revised the manuscript. $Z Z$ and GL drafted the manuscript. ML, ML and MX contributed to the revision of the manuscript. All authors read and approved the final manuscript.

\section{Acknowledgements}

No applicable

\section{References}

1. Nelen SD, Verhoeven RHA, Lemmens VEPP, de Wilt JHW, Bosscha K. Increasing survival gap between young and elderly gastric cancer patients. Gastric Cancer. 2017;20(6):919-28. doi:10.1007/s10120017-0708-7.

2. Li WY, Han Y, Xu HM, et al. Smoking status and subsequent gastric cancer risk in men compared with women: a meta-analysis of prospective observational studies. BMC Cancer. 2019;19(1):377.

Published 2019 Apr 24. doi:10.1186/s12885-019-5601-9.

3. Rawla P, Barsouk A. Epidemiology of gastric cancer: global trends, risk factors and prevention. Prz Gastroenterol. 2019;14(1):26-38. doi:10.5114/pg.2018.80001.

4. Mukhopadhyay A, Shinde A, Naik R. Ovarian cysts and cancer in pregnancy. Best Pract Res Clin Obstet Gynaecol. 2016;33:58-72.

5. Engelberth SA, Hempel N, Bergkvist M. Development of nanoscale approaches for ovarian cancer therapeutics and diagnostics. Crit Rev Oncog. 2014;19(3-4):281-315.

doi:10.1615/critrevoncog.2014011455. 
6. Sayedur RM, Al-Sibai MH, $\mathrm{i}^{1} / 4^{2}$ ahman J,et al. Ovarian carcinoma associated with pregnancy. A review of 9 cases. Acta Obstet Gynecol Scand. 2002;81(3):260-4.

7. Yin B, Li W, Cui Y, et al. Value of diffusion-weighted imaging combined with conventional magnetic resonance imaging in the diagnosis of thecomas/fibrothecomas and their differential diagnosis with malignant pelvic solid tumors. World J Surg Oncol. 2016;14(1):5. Published 2016 Jan 8. doi:10.1186/s12957-015-0760-x.

8. Song MJ, Park YS, Song HJ, et al. Prognosis of Pregnancy-Associated Gastric Cancer: An Age-, Sex-, and Stage-Matched Case-Control Study. Gut Liver. 2016;10(5):731-8. doi:10.5009/gnl15323.

9. Kulbe H, Otto R, Darb-Esfahani S, et al. Discovery and Validation of Novel Biomarkers for Detection of Epithelial Ovarian Cancer. Cells. 2019;8(7):713. Published 2019 Jul 12. doi:10.3390/cells8070713.

10. Kelten C, Boyaci C, Leblebici C, et al. Malignant Phyllodes Tumor Including Aneurysmal Bone CystLike Areas in Pregnancy - a Case Report and Review of the Literature. Breast Care (Basel). 2016;11(4):291-4. doi:10.1159/000448236.

11. Kim SH, Abd Halim SR, Siddiqui N, Park WH. Disseminated cancer in pregnancy: krukenberg tumour. Case Rep Obstet Gynecol. 2014;2014:216969. doi:10.1155/2014/216969.

12. Zhang J, Peng P, Cao D, Yang J, Shen K. Clinicopathological features and maternal and foetal management of pregnancy-complicating Krukenberg tumours. Mol Clin Oncol. 2020;12(6):581-7. doi:10.3892/mco.2020.2025.

13. Cormedi MCV, Katayama MLH, Guindalini RSC, Faraj SF, Folgueira MAAK. Survival and prognosis of young adults with gastric cancer. Clinics (Sao Paulo). 2018;73(suppl 1):e651s. Published 2018 Sep 21. doi:10.6061/clinics/2018/e651s.

\section{Figures}




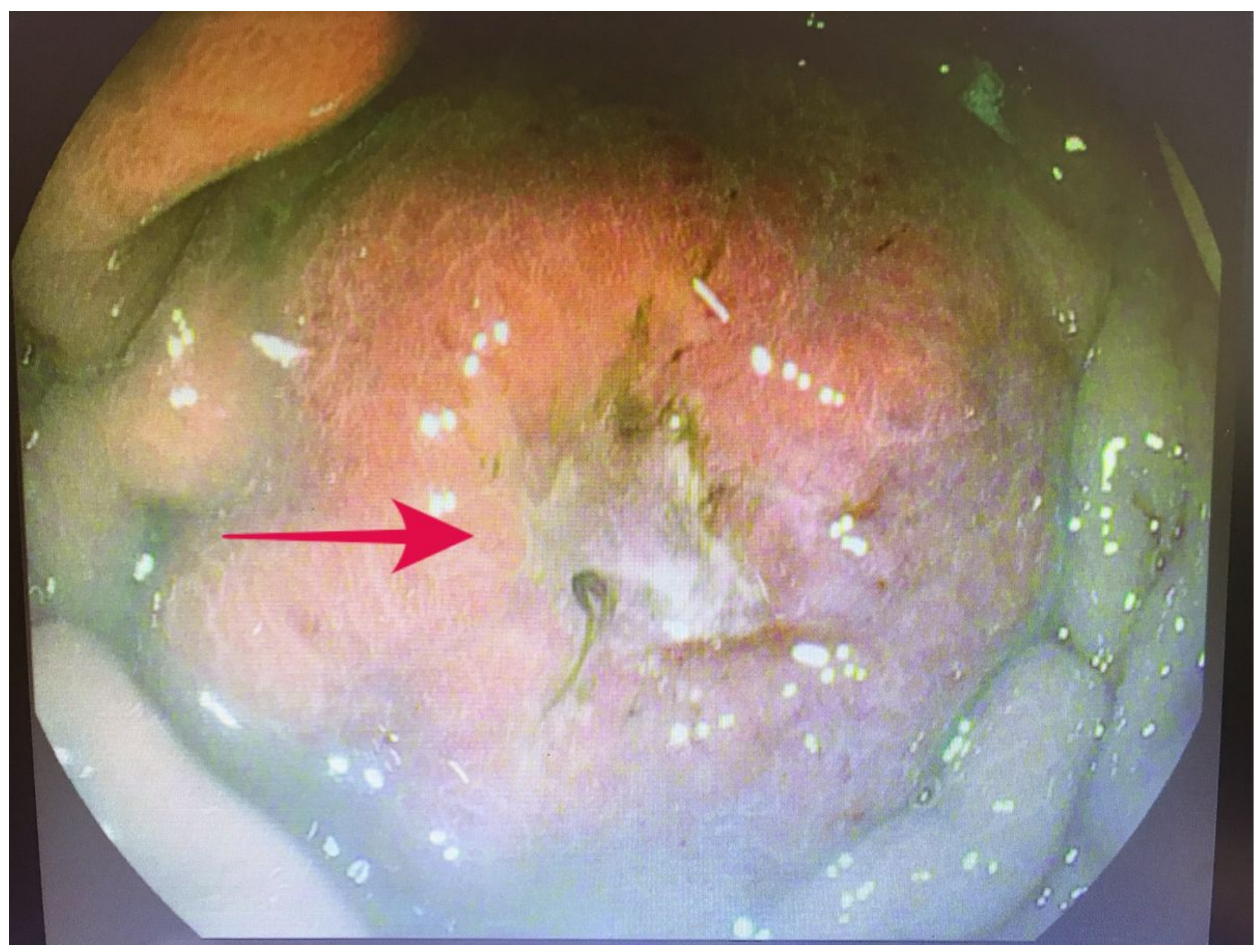

Figure 1

The gastric mucosa was rough, and there was an ulcer with a diameter of about $1.0 \mathrm{~cm}$ in the greater curvature of the gastric body. 


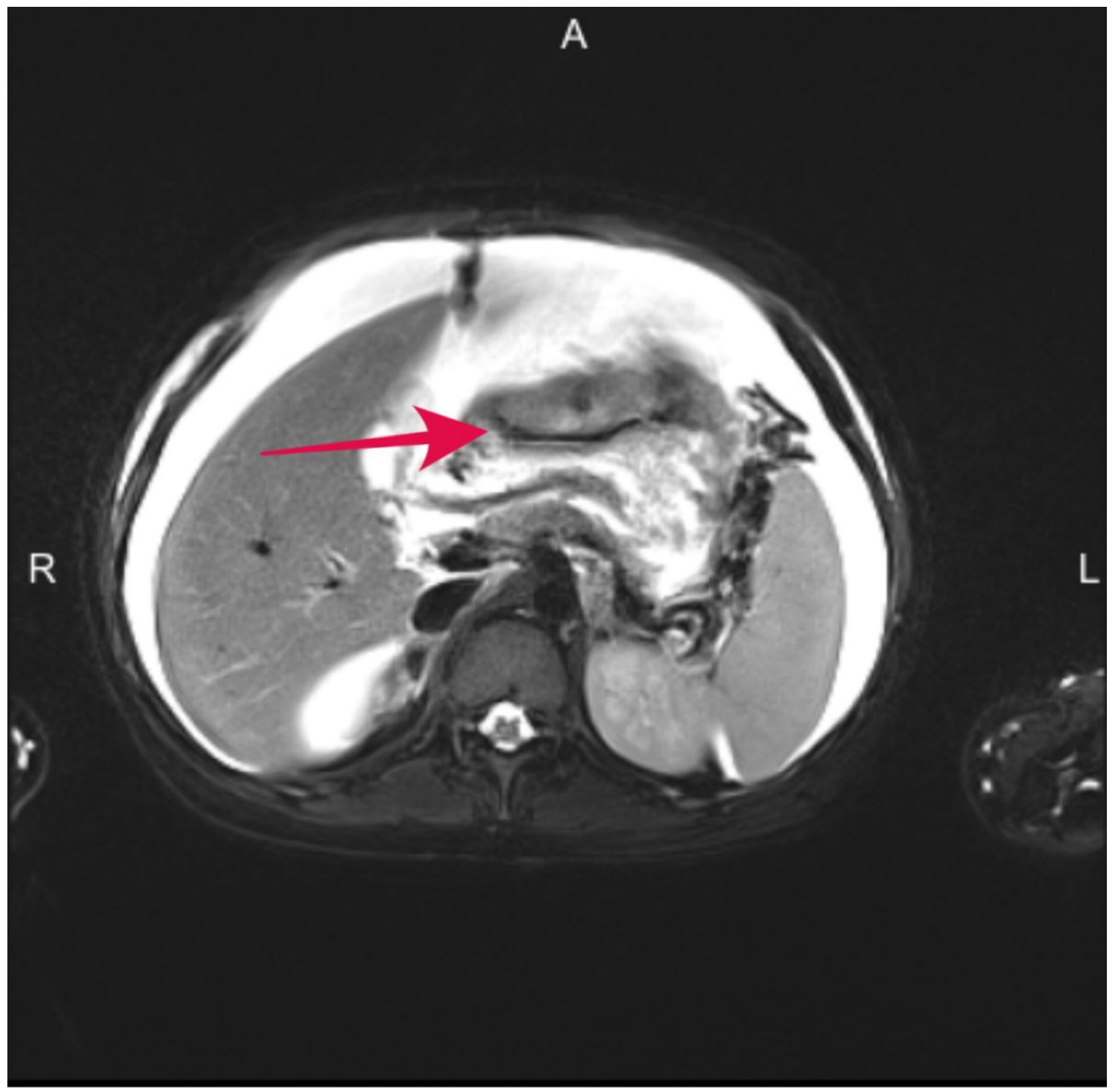

\section{Figure 2}

T2WI fat suppression sequence: Irregular masses of long T 1 and long T 2 signal shadows were found in the body of the stomach. DWI showed a slightly high signal with a clear edge. 


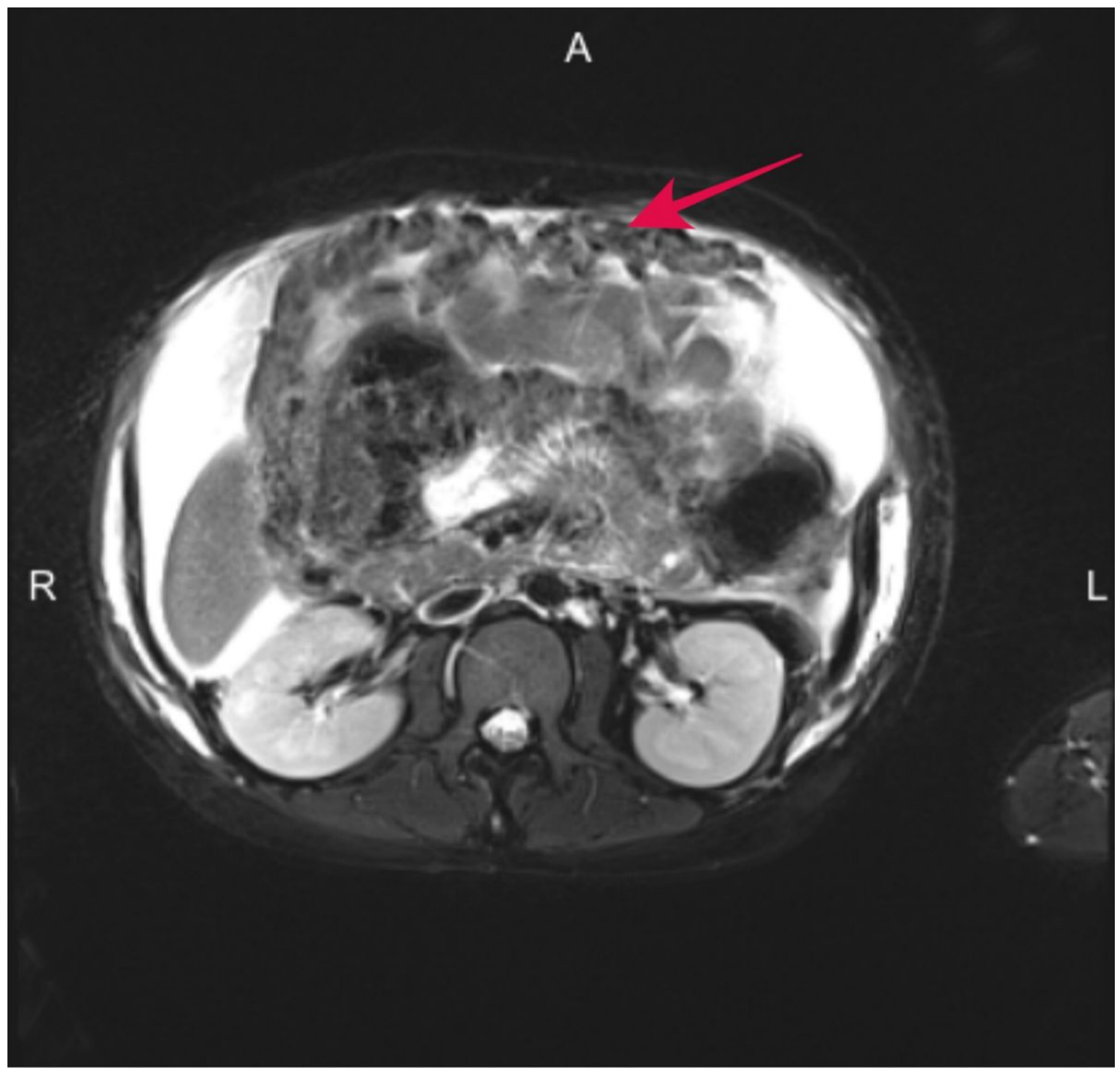

\section{Figure 3}

T2WI fat suppression sequence: The greater omentum was thickened irregularly, presented multiple nodules and strips, with short T1 and long T2 signals. 

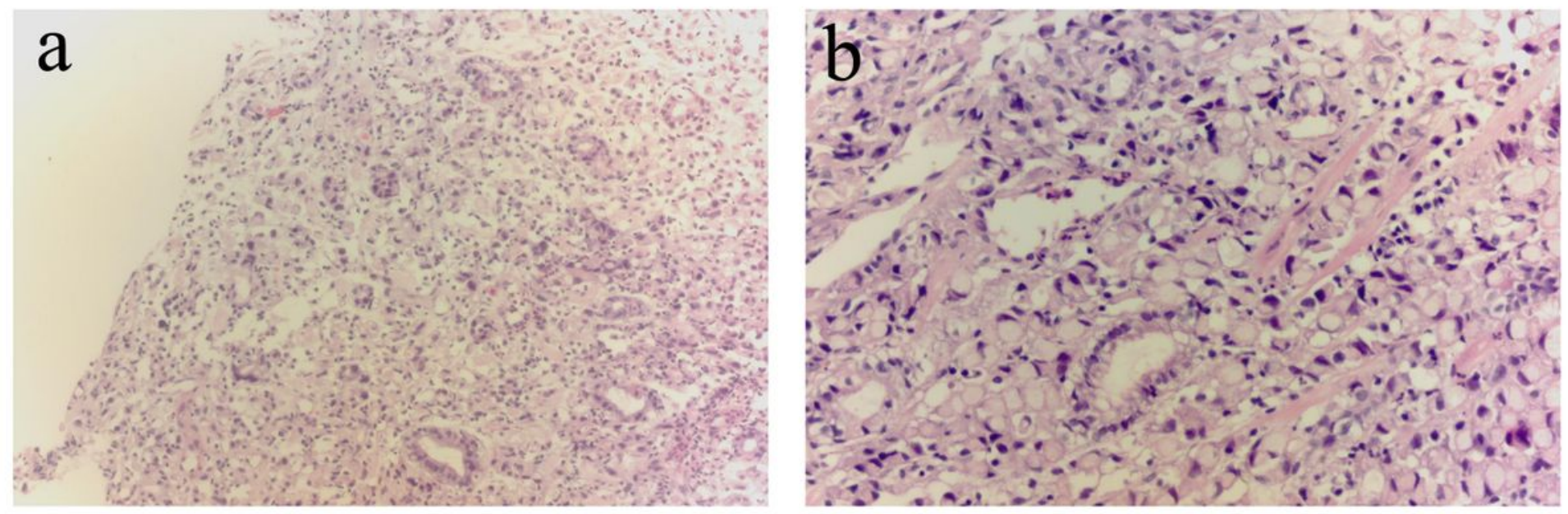

\section{Figure 4}

a: Tumor cells were mainly located in the surface layer and lamina propria of the mucosa, and there was no muscularis mucosa. The tumor cells are distributed in sheets and grow infiltrating, most of which were signet ring-like. b: Most of the tumor cells were signet ring-like. 